\title{
A Novel Visibility Restoration Technique for Hazy Videos Captured in Real - World Weather Conditions
}

\author{
Meenu Ravi ${ }^{1}$, Devi Murali ${ }^{2}$ \\ ${ }^{1}$ M .Tech student, Department of Communication Engineering, Sree Buddha College of Engineering for Women,
} Elavumthitta, Kerala, India

${ }^{2}$ Assistant Professor, Department of Electronics and Communication Engineering, Sree Buddha College of Engineering for Women, Elavumthitta, Kerala, India

\begin{abstract}
The visibility of outdoor videos were seriously degraded under hazy, foggy and sandstorm weather conditions, which will affect the computer vision applications, such as outdoor object recognition systems, obstacle detection systems, video surveillance systems, and intelligent transportation systems. This paper proposes a novel method for restoring the visibility of degraded videos that uses a combination of three major modules: 1) a depth estimation (DE) module; 2) a color analysis (CA) module; and 3) a visibility restoration (VR) module. This visibility restoration approach is based on the conjunctive utilization of the median filter and guided joint bilateral filter operation, the adaptive gamma correction technique, the gray world assumption, and the dark channel prior method. The proposed DE module takes advantage of the median filter technique and adopts adaptive gamma correction technique. By doing so, halo effects can be avoided in videos with complex structures, and can achieve an effective estimate of the transmission map. The proposed CA module is based on the gray world assumption and analyzes the color features of each input hazy frames. Subsequently, the VR module uses the adjusted transmission map and the color-correlated information to mend the color distortion in variable scenes captured during inclement weather conditions. The proposed method will successfully be applied to videos; hence we can use this algorithm in even real time video applications.
\end{abstract}

Keywords: Dark Channel Prior, Depth Estimation, Median Filter, Visibility Restoration.

\section{Introduction}

The images or videos captured in inclement weather conditions is often degraded due to the presence of the turbid medium (e.g., particles and water droplets) in the atmosphere that will absorb and scatter light between the digital camera and the captured object. Haze removal (or dehazing) is highly desired in consumer or computational photography and computer vision applications. Numerous haze removal techniques have been proposed by which to improve visibility in hazy videos. [2] proposed a method that dehaze the videos using dark channel method along with the use of guided filter. Currently, the method of [2] is generally considered to be the best haze removal approach for videos. However, the efficacy of haze removal may change in response to varied weather conditions and scene objects in realistic environments. In particular, the method proposed in [2] cannot adequately deal with color distortions and complex structures. In these situations, restored videos will feature color shift and artifact effects. Therefore, we propose a novel VR approach for videos that is based on an existing method which mainly focuses on image dehazing [1]. The proposed method is based on the conjunctive utilization of median filter and joint bilateral filter operation, adaptive gamma correction technique, gray world assumption, and the dark channel prior method. In this method, firstly the hazy video is divided into frames. The number of frames depends on the size of the video. Then each frame can be considered as similar to a single hazy image, and on each of those hazy images our method is applied. Finally all the haze free frames were combined to get the dehazed output video. With the target of haze removal, multiple image haze removal methods [6] were first introduced. In the multiple image haze removal method, two or more images of the same scene were taken. This strategy increases the number of known variables, at the same time it brings more unknown, so special settings must be needed to avoid too many unknown being introduced. The dichromatic method [7], takes multiple images of the same scene, under different atmospheric conditions. The main problem of this method is that weather may remain unchanged in several minutes or even hours. Polarization based method [8] is another multiple image haze removal technique. Its main problem is its settings, that is, capturing two strictly aligned polarized images is troublesome. To overcome the limitation of multiple image haze removal another strategy, single image haze removal $[1,4]$ introduced. It is based on some assumption or knowledge. This strategy become more popular since, it requires only one image. Our new method introduces three major modules in video dehazing. The key features of our proposed method are as follows.

1) In order to avoid generation of halo effects and insufficient estimation of the transmission map, the proposed depth estimation (DE) module contains two major procedures that take advantage of the median filter to preserve edge information and a guided joint bilateral filtering is used to refine the median filter output. The adaptive gamma correction technique is employed to adjust the intensity of the transmission map.

2) Next, the proposed color analysis (CA) module uses the gray world assumption to analyze the color characteristics of

Volume 5 Issue 6, June 2016 www.ijsr.net 


\section{International Journal of Science and Research (IJSR) \\ ISSN (Online): 2319-7064}

Index Copernicus Value (2013): 6.14 | Impact Factor (2015): 6.391

input images. The obtained color information can express the variation range of RGB distribution and thereby circumvents color distortion problems.

3) The proposed VR module uses the adjusted transmission map and color-correlated information produced, respectively, by the DE and CA modules to recover high-quality haze-free image or frames. Finally by combining all those frames, we get final video as haze free one.

The remainder of this paper is organized as follows. In Section 2, we describe the proposed haze removal method for videos. Section 3 presents the experimental results and the discussions based on it. Finally, the conclusion is presented in Section 4.

\section{Proposed Method}

In this section, we propose a novel dehazing method in order to restore the visibility of hazy videos captured during inclement weather conditions. Here we explain how our proposed method works. We know that a video can be divided into different frames and each frame is equivalent to a single image. So our hazy video is first divided into different number of hazy frames and upon each such frame our method for haze removal is applied to obtain haze free frames. Finally by combining all those frames a haze free video is obtained.

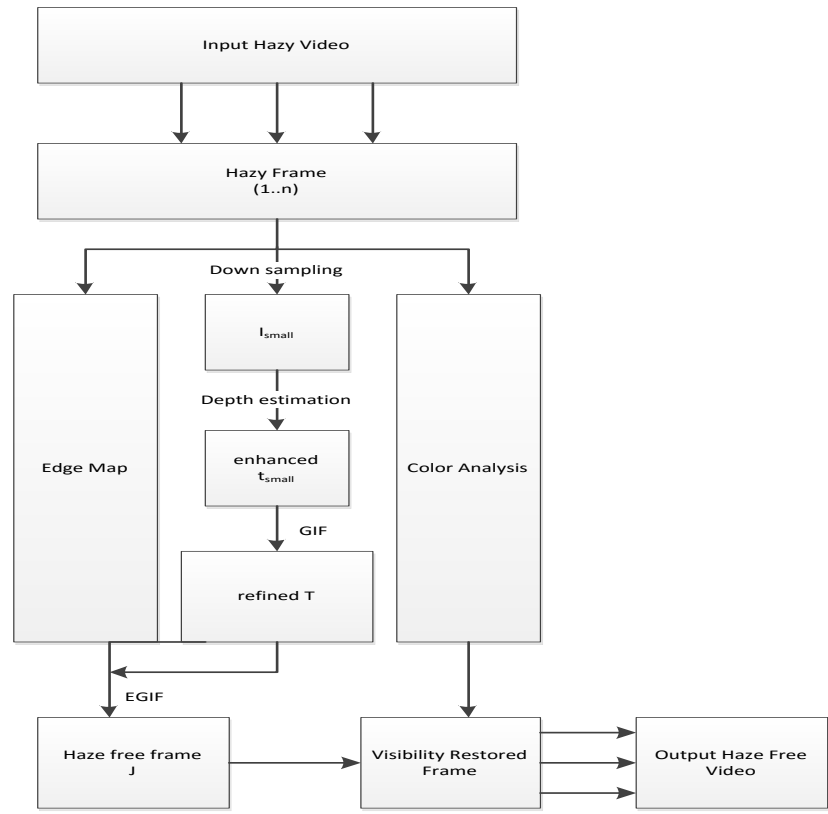

Figure 1: Flowchart of our proposed VR approach

The flowchart of the proposed VR algorithm is shown in Figure 1. The method need to apply on each hazy frame is described in detail below:

\subsection{Haze Image Equation}

In computer vision and graphics, the optical model widely used to describe the formation of a degraded hazy image is:

$$
I(x)=J(x) t(x)+A(1-t(x))
$$

where $I(x)$ is the intensity of the observed hazy image, $J(x)$ is the scene radiance, $A$ is the global atmospheric light, and $t(x)$ is the transmission map that represents the portion of light, which is not scattered and subsequently received by the camera without any attenuation. On the righthand side of (1), the first term $J(x) t(x)$ is called the direct attenuation; the second term $A(1-t(x))$ is called airlight. Direct attenuation describes the decay of scene radiance which depends on the medium and scene depth, while airlight represents the scattering of light which leads to the shift of the scene colors.

\subsection{Dark Channel Prior}

In order to restore the visibility of degraded images, He et al. [4] presents a dark channel prior method to estimate scene depth in a single image. The dark channel prior method is based on the key observation that an outdoor haze free image exhibits at least one color channel that has some pixels whose intensity are very low and close to zero. Thus, the dark channel $J^{\text {dark }}$ of the hazy image can be expressed as:

$$
J^{\text {dark }}(x)=\min _{y \in \Omega(x)}\left(\min _{c \in\{r, g, b\}} J^{c}(y)\right)
$$

Where $J^{c}$ is a color channel of the RGB image $J$, $\min _{c \in\{r, g, b\}}$ is the minimum value of the color channel, $\Omega(x)$ is a local patch centered at location $x$, and $\underset{y \in \Omega(x)}{\min }$ is a minimum filter. While taking the concept of dark channel, if $J$ is an outdoor haze-free image, the corresponding intensity of dark channel $J^{\text {dark }}$ is low and close to zero.

\subsection{Estimating the Atmospheric Light}

The highest intensity pixels among the $0.1 \%$ brightest pixels that were chosen from the dark channel prior of input hazy image is selected as the atmospheric $\operatorname{light} A$. Since the brightest portion of a dark channel appears as most opaque, it is considered as the global atmospheric light.

\subsection{Estimating the Transmission Map}

We can easily estimate the transmission value by normalizing (1) by the atmospheric light $A$ as:

$$
\frac{I^{c}(x)}{A^{c}}=t(x) \frac{J^{c}(x)}{A^{c}}+(1-t(x))
$$

The method of dark channel prior is applied on both sides of (3) as:

$$
\begin{aligned}
\min _{y \in \Omega(x)}\left(\min _{c \in\{r, g, b\}} \frac{I^{c}(y)}{A^{c}}\right)= & t(x) \min _{y \in \Omega(x)}\left(\min _{c \in\{r, g, b\}} \frac{J^{c}(y)}{A^{c}}\right)+ \\
& 1-t(x)
\end{aligned}
$$

The dark channel prior of an outdoor haze free image will be close to zero, as illustrated by: 


\section{International Journal of Science and Research (IJSR) \\ ISSN (Online): 2319-7064}

Index Copernicus Value (2013): 6.14 $\mid$ Impact Factor (2015): 6.391

$$
\min _{y \in \Omega(x)}\left(\min _{c \in\{r, g, b\}} \frac{J^{c}(y)}{A^{c}}\right)=0
$$

The transmission value can be derived from (4) and (5) as:

$$
t(x)=1-\min _{y \in \Omega(x)}\left(\min _{c \in\{r, g, b\}} \frac{I^{c}(y)}{A^{c}}\right)
$$

Unfortunately, if we remove the haze completely, the results may seem unnatural. Thus, He et al. [4] add a constant $\omega$ $(0<\omega<1)$ into $(6)$ as

$$
t(x)=1-\omega \min _{y \in \Omega(x)}\left(\min _{c \in\{r, g, b\}} \frac{I^{c}(y)}{A^{c}}\right)
$$

where $\omega$ is set to 0.95 .

\subsection{Depth Estimation (DE) Module}

Regarding the dark channel prior technique, two prominent problems were existed: 1) Halo effect generation and 2) estimation of insufficient transmission map. In order to circumvent these problems a DE module is proposed [1]. It is used to estimate the transmission map of a hazy image directly and is based primarily on the dark channel prior technique. However, the DE module circumvents the aforementioned problems using a refined transmission procedure and an enhanced transmission procedure.

\subsubsection{Refined Transmission}

Since dark channel prior utilizes minimum filter, there will be loss of edge information while estimating the transmission map. Hence, a refined transmission procedure is proposed that utilizes a median filter technique to preserve edge information of input hazy images and thereby avoid generation of halo effects. A nonlinear filtering operation is performed by the median filter that can effectively suppress impulsive noise components while preserving edge information. The edge information $D(x)$ in detail can be calculated as:

$$
\begin{aligned}
D(x)= & \omega\left(\min \left(\operatorname{median}_{y \in \Omega(x)}(W(y)), W(x)\right)\right)- \\
& \min _{y \in \Omega(x)}(W(y))
\end{aligned}
$$

where $\omega$ is set to $0.95, \Omega(x)$ is a local patch centered at position $x$, median is a median filter, and min is a minimum filter. $W(x)$ is an independent pixel at position $x$, and $W(y)$ is each independent pixel of the corresponding patch $\Omega(x)$. The refined transmission $t_{r}(x)$ can be obtained via the edge information as:

$$
t_{r}(x)=t(x)-D(x)
$$

where $t(x)$ is the transmission map.

\subsubsection{Guided Joint Bilateral Filtering}

A guided joint bilateral filtering is used to refine the median filter output. By means of a nonlinear combination of nearby pixel values bilateral filtering smoothes images while preserving the edge information. The bilateral filter computes the filter output as a weighted average of neighboring pixels. It is generalized to joint bilateral filter in which the weights are computed from another guidance image rather than the filter input. The joint bilateral filter is usually favored in such situations where the filter input is not reliable to provide edge information. The filtering process can be generally defined by a guidance image $I$, an input image $p$, and an output image $q$.

The filtering output at a pixel $i$ is expressed as a weighted average of:

$$
q_{i}=\sum_{j} W_{i j}(I) p_{j}
$$

where $i$ and $j$ are pixel indexes. The filter kernel $W_{i j}$ is a function of the guidance image $I$ and is independent of $p$. This filter is linear with respect to $p$. The best example of such a filter is the joint bilateral filter. The bilateral filtering kernel $W_{b f}$ is given by:

$$
\begin{aligned}
W_{i j}^{b f}(I)= & \frac{1}{K_{i}} \exp \left(-\frac{\left|x_{i}-x_{j}\right|^{2}}{\sigma_{s}^{2}}\right) \\
& \exp \left(-\frac{\left|I_{i}-I_{j}\right|^{2}}{\sigma_{r}^{2}}\right)
\end{aligned}
$$

where $x$ is the pixel coordinate, and $K_{i}$ is a normalizing parameter to ensure that $\sum_{j} W_{i j}^{b f}=1$. The parameters $\sigma_{s}$ and $\sigma_{r}$ adjust the spatial and the range similarity respectively. When $I$ and $p$ are identical, the joint bilateral filter becomes equivalent to original bilateral filter.

\subsubsection{Enhanced Transmission}

For images captured during inclement weather conditions the dark channel prior technique always estimates an insufficient transmission map. This is due to the presence of at least one low intensity color channel value in hazy images. An adaptive gamma correction technique is used to adjust the intensity of the transmission map in order to obtain optimum haze removal results. The enhanced transmission form $t_{e}(x)$ is derived via the adaptive gamma correction operate as:

$$
\begin{gathered}
t_{e}(x)=\left(X_{\max }\right)\left(\frac{t_{r}(x)}{X_{\max }}\right)^{\gamma} \\
\gamma= \begin{cases}1+\left(\frac{t}{X_{\max }}\right) & \text { if } \mathrm{t} \geq \mathrm{T} \\
1 & \text { if } \mathrm{t}<\mathrm{T}\end{cases}
\end{gathered}
$$

where $X_{\max }$ is the maximum intensity of the input image, $t_{r}(x)$ is the refined transmission map, $\gamma$ is a varying adaptive parameter, and $t$ is an intensity value when cumulative probability density (CDF) is equal to 0.1 . The adaptive threshold value $T$ is empirically set to 120 .

\section{Volume 5 Issue 6, June 2016 www.ijsr.net}




\section{International Journal of Science and Research (IJSR) \\ ISSN (Online): 2319-7064}

Index Copernicus Value (2013): 6.14 | Impact Factor (2015): 6.391

\subsection{Color Analysis (CA) Module}

The images and videos captured in inclement weather conditions usually exhibit serious color distortion problems. It is because the atmospheric particle absorbs particular portions of the color spectrum. In order to solve this problem, the CA module [1] utilizes the gray world assumption to determine whether or not the average intensities of the each color channel are equal. The average intensities of the RGB color channels are calculated by:

$$
a v g_{c}=\frac{\sum_{i=1}^{M} \sum_{j=1}^{N} I^{c}(i, j)}{M N}, \quad \text { for } c \in\{r, g, b\}
$$

where the size of the observed image is $M N$ pixels.

The color difference value $d^{c}$ for RGB color channels are calculated using the average intensity of each color channel as:

$$
d^{c}=a v g_{r}-a v g_{c} \quad, \quad \text { for } c \in\{r, g, b\}
$$

\subsection{Visibility Restoration (VR) Module}

The VR module [1] calculates the scene radiance via the enhanced transmission map and the color difference value as:

$$
J^{c}(x)=\frac{I^{c}(x)-\left(A^{c}-d^{c}\right)}{\max \left(t_{e}(x), t_{0}\right)}+\left(A^{c}-d^{c}\right)
$$

where $J(x)$ is the scene radiance, $I(x)$ is the intensity of the observed hazy image, $A$ is the global atmospheric light, $d^{c}$ is a color difference value, $t_{e}(x)$ is the enhanced transmission map, and $t_{0}$ is typically set to 0.1 . Hence, we can employ (16) to effectively recover the visibility of a degraded hazy image. After performing all the above operations, we get a collection of haze free frames. By combining all those clear frames, we get the final video as a haze free one.

\section{Results and Discussions}

In this section, we are discussing about the experimental results and its comparison with previous methods. The Figure 2(a) shows a single input hazy image and Figure 2(b) shows the corresponding haze free image. From the appearance itself we can distinguish the difference between two images. Figure 3 (a) shows the input hazy image and its

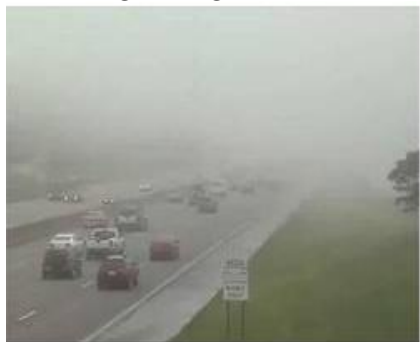

(a)

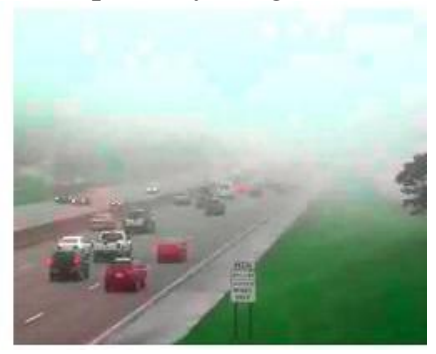

(b)
Figure 2: Haze removal results. (a) Input hazy frame and (b) Haze free frame. corresponding dark channel and enhanced transmission map are shown in Figure 3 (b) and (c). The brightest pixel from the dark channel is chosen as the global atmospheric light. From which we can easily estimate enhanced transmission and recover haze free image.

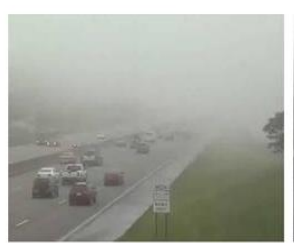

(a)

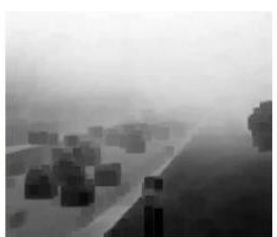

(b)

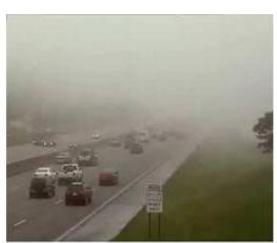

(c)
Figure 3: (a) Input hazy image. (b) Dark channel (c) Enhanced transmission map.

The resultant dehazed video representation is shown in Figure 4.
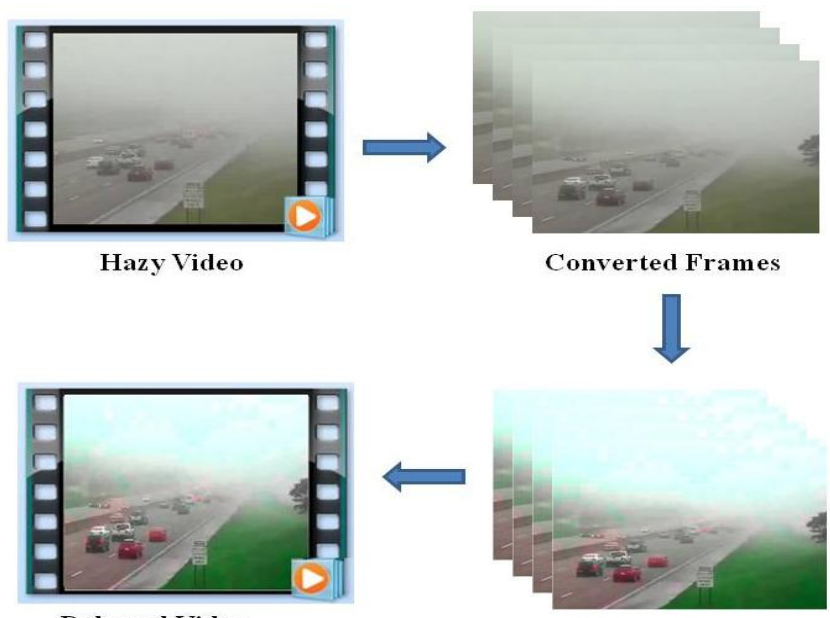

Dehazed Video

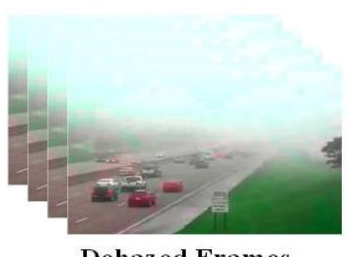

Dehazed Frames

Figure 4: Video Dehazing.

Figure 5 shows, the base output, the outputs obtained by using various filters such as imfilter, hybrid median filter and guided filter and the output of our proposed method when applied on the hazy image. They show the improvement by using different filters. The input hazy image becomes clearer by the effect of each filter. From the appearance itself it is clear that a better output is obtained by our proposed method. The differences in the effect of each output can be analyzed by a parameter called visibility metric value [2]. Analyses of visibility metric values are shown in Figure 5 and Table 1: 


\section{International Journal of Science and Research (IJSR) \\ ISSN (Online): 2319-7064}

Index Copernicus Value (2013): 6.14 | Impact Factor (2015): 6.391

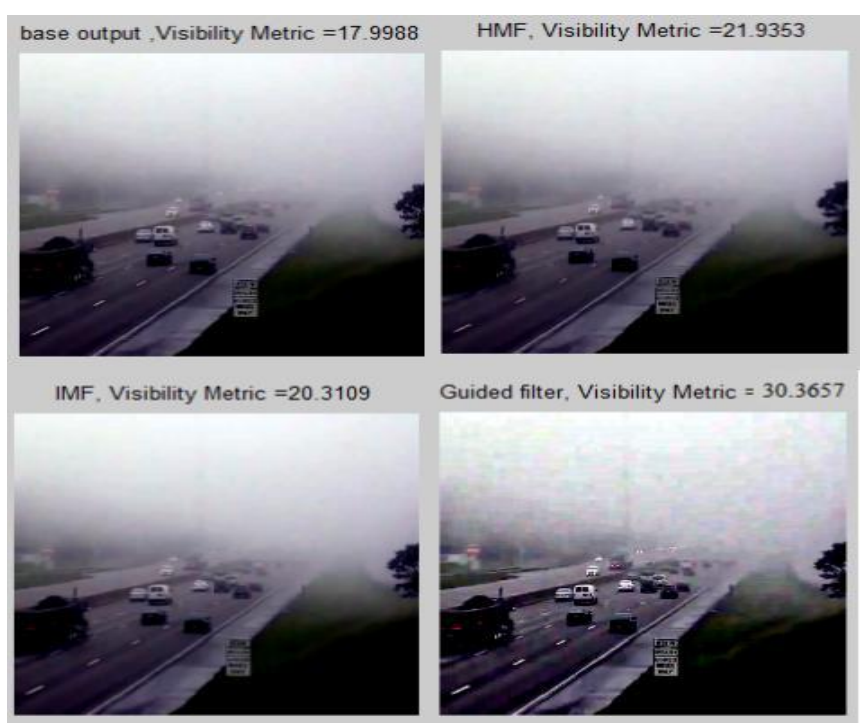

Proposed Method, Visibility Metric $=36.1030$

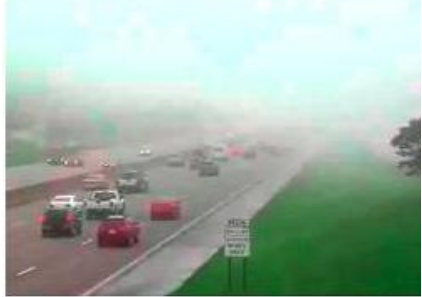

Figure 5 : Visibility Metric for different results.

Table 1: Comparison of Visibility Metric value for different Methods

\begin{tabular}{|c|c|}
\hline Methods & Visibility Metric Value \\
\hline Base Output & 17.9988 \\
\hline HM filter & 21.9353 \\
\hline IM filter & 20.3109 \\
\hline Guided filter & 30.3657 \\
\hline Proposed Method & 36.1030 \\
\hline
\end{tabular}

From Table 1 and from Figure 5, it is clear that our proposed method has the highest visibility metric value, so it provides better enhancement and results.

\section{Conclusion}

This paper proposed a method for video dehazing. Existing methods mainly focus on image dehazing. But here we explain a video dehazing technique. The proposed approach utilizes a combination of three major modules: 1) a Depth Estimation module; 2) a Color Analysis module; and 3) a Visibility Restoration module. The experimental results produced by this method was evaluated and compared with previous results by an image quality parameter called Visibility Metric Value. This analysis shows the efficacy of our proposed VR technique. Not only this method circumvents significant problems regarding color distortion and complex structure, but also produces high-quality hazefree video more effectively.

\section{Acknowledgment}

I would like to express profound gratitude to our Head of the Department, Prof. Cherian Schariah, for his encouragement and for providing all facilities for my work. I express my highest regard and sincere thanks to my guide, Asst. Prof. Ms. Devi Murali., who provided me the necessary guidance and serious advice for my work.

\section{References}

[1] Shih-Chia Huang, Bo-Hao Chen, and Wei-Jheng Wang, "Visibility Restoration of Single Hazy Images Captured in Real-World Weather Conditions," IEEE Trans. Circuits Syst. Video Technol., vol. 24, no. 10, Oct. 2014.

[2] Shari Thomas, Lintu Liz Thomas, Mathews M, Tonu James, "Restoration of Hazy Videos Using Dark Channel Approach and Guided Filtering," IJERT, vol. 3 Issue 5, May. 2014.

[3] Zheqi Lin, Xuansheng Wang, "Dehazing for Image and Video Using Guided Filter," Open Journal of Applied Sciences, 2012 World Congress on Engg. and Technol.

[4] Kaiming He, Jian Sun, and Xiaoou Tang, "Single Image Haze Removal Using Dark Channel Prior," IEEE Trans. on Pattern Analysis and Machine Intelligence, vol. 33, no. 12, Dec. 2011.

[5] R. Fattal, "Single image dehazing," in Proc. ACM SIGGRAPH, 2008.

[6] S. K. Nayar and S. G. Narasimhan, "Vision in bad weather," in Proc 7th IEEE ICCV, vol. 2. Jun. 1999, pp. $820-827$.

[7] S.G. Narasimhan and S.K. Nayar, "Vision and the Atmosphere" Intl J Computer Vision, vol. 48, pp. 233 254, 2002.

[8] Y.Y. Schechner, S.G. Narasimhan, and S.K. Nayar, "Instant Dehazing of Images Using Polarization" Proc. IEEE Conf. Computer Vision and Pattern Recognition, vol. 1, pp. 325-332, 2001.

[9] www.mathworks.in

\section{Author Profile}

Meenu Ravi received the B-Tech degree in Electronics and Communication Engineering from M.G University, Kerala at Sree Buddha College of Engineering for Women in 2014. And now she is pursuing her $\mathrm{M}$-Tech degree in Communication Engineering under the same university in Sree Buddha College of Engineering for Women.

Devi Murali working as an Assistant Professor in Department of Electronics and Communication, Sree Buddha College of Engineering for Women, Elavumthitta, Pathanamthitta. 